\title{
Effect of early intensive multifactorial therapy compared with routine care on self-reported health status, general well-being, diabetes-specific quality of life and treatment satisfaction in screen-detected type 2 diabetes mellitus patients (ADDITION-Europe): a cluster-randomised trial
}

\author{
Maureen Van den Donk • Simon J. Griffin • Rebecca K. Stellato • Rebecca K. Simmons • \\ Annelli Sandbæk • Torsten Lauritzen • Kamlesh Khunti • Melanie J. Davies • \\ Knut Borch-Johnsen • Nicholas J. Wareham • Guy E. H. M. Rutten
}

Received: 22 April 2013 / Accepted: 13 July 2013 / Published online: 20 August 2013

(C) The Author(s) 2013. This article is published with open access at Springerlink.com

\begin{abstract}
Aims/hypothesis The study aimed to examine the effects of intensive treatment (IT) vs routine care (RC) on patientreported outcomes after 5 years in screen-detected diabetic patients.

Methods In a pragmatic, cluster-randomised, parallel-group trial, 343 general practices in Denmark, Cambridge and Leicester (UK) and the Netherlands were randomised to screening for type 2 diabetes mellitus plus IT of multiple risk factors in people 40-69 years without known diabetes ( $n=1,678$ patients) or screening plus RC ( $n=1,379$ patients). Practices were randomised in a 1:1 ratio according to a computer-generated list. Diabetes mellitus was diagnosed according to WHO
\end{abstract}

Electronic supplementary material The online version of this article (doi:10.1007/s00125-013-3011-0) contains peer-reviewed but unedited supplementary material, which is available to authorised users.

M. Van den Donk · R. K. Stellato • G. E. H. M. Rutten ( $\triangle)$

Julius Center for Health Sciences and Primary Care,

University Medical Center Utrecht, PO Box 85500,

3508 GA Utrecht, the Netherlands

e-mail: g.e.h.m.rutten@umcutrecht.nl

S. J. Griffin • R. K. Simmons • N. J. Wareham

MRC Epidemiology Unit, University of Cambridge, Cambridge, UK

A. Sandbæk $\cdot$ T. Lauritzen

Department of Public Health, Section of General Practice,

University of Århus, Århus, Denmark

K. Khunti • M. J. Davies

Diabetes Research Unit, University of Leicester, Leicester, UK

K. Borch-Johnsen

Holbaek Hospital, Holbaek, Denmark criteria. Exclusions were: life expectancy $<1$ year, housebound, pregnant or lactating, or psychological or psychiatric problems. Treatment targets for IT were: $\mathrm{HbA}_{1 \mathrm{c}}<7.0 \%$ ( $53 \mathrm{mmol} / \mathrm{mol}$ ), $\mathrm{BP} \leq 135 / 85 \mathrm{mmHg}$, cholesterol $<5 \mathrm{mmol} / \mathrm{l}$ in the absence of a history of coronary heart disease and $<4.5 \mathrm{mmol} / \mathrm{l}$ in patients with cardiovascular $(\mathrm{CV})$ disease; prescription of aspirin to people taking antihypertensive medication and, in cases of CV disease or BP $>120 / 80 \mathrm{mmHg}$, ACE inhibitors were recommended. After 2003, the treatment algorithm recommended statins to all patients with cholesterol of $\geq 3.5 \mathrm{mmol} / 1$. Outcome measures were: health status (Euroqol 5 Dimensions [EQ-5D]) at baseline and at follow-up; and health status (36-item Short Form Health Survey [SF-36] and Euroquol Visual Analogue Scale [EQVAS]), well-being (12-item Short Form of the Well-Being Questionnaire), diabetes-specific quality of life (Audit of Diabetes-Dependent Quality of Life) and satisfaction with diabetes treatment (Diabetes Treatment Satisfaction Questionnaire) at follow-up. At baseline, standardised selfreport questionnaires were used to collect information. Questionnaires were completed at the same health assessment visit as the anthropometric and biochemical measurements. The patients and the staff assessing the outcomes were unaware of the group assignments. Participants were followed for a mean of 5.7 years. Outcome data were available for 1,250 participants in the intensive treatment group (74\%) and 967 participants in the routine care group (70\%). The estimated differences in means from the four centres were pooled using random effects meta-analysis. Baseline EQ-5D level was used as a covariate in all analyses.

Results EQ-5D values did not change between diagnosis and follow-up, with a median (interquartile range) of 0.85 
$(0.73-1.00)$ at baseline and $0.85(0.73-1.00)$ at 5 year followup. Health status, well-being, diabetes-specific quality of life and treatment satisfaction did not differ between the intensive treatment and routine care groups. There was some heterogeneity between centres ( $I^{2}$ being between 13\% [SF-36 physical functioning] and $73 \%$ [EQ-VAS]).

Conclusions/interpretation There were no differences in health status, well-being, quality of life and treatment satisfaction between screen-detected type 2 diabetes mellitus patients receiving intensive treatment and those receiving routine care. These results suggest that intensive treatment does not adversely affect patient-reported outcomes.

Trial registration number ClinicalTrials.gov NCT00237549

Funding ADDITION-Denmark was supported by the National Health Services, the Danish Council for Strategic Research, the Danish Research Foundation for General Practice, Novo Nordisk Foundation, the Danish Centre for Evaluation and Health Technology Assessment, the Diabetes Fund of the National Board of Health, the Danish Medical Research Council and the Aarhus University Research Foundation. In addition, unrestricted grants from pharmaceutical companies were received. ADDITION-Cambridge was supported by the Wellcome Trust, the Medical Research Council, the NIHR Health Technology Assessment Programme, National Health Service R\&D support funding and the National Institute for Health Research. SJG received support from the Department of Health NIHR grant funding scheme. ADDITION-Leicester was supported by Department of Health, the NIHR Health Technology Assessment Programme, National Health Service R\&D support funding and the National Institute for Health Research. ADDITIONNetherlands was supported by unrestricted grants from Novo Nordisk, Glaxo Smith Kline and Merck, and by the Julius Center for Health Sciences and Primary Care, University Medical Center, Utrecht.

Keywords Diabetes screening $\cdot$ Health status $\cdot$ Intensive treatment · Patient-reported outcome measures · Primary care . Quality of life · Treatment satisfaction · Type 2 diabetes mellitus

\begin{tabular}{|c|c|}
\hline \multicolumn{2}{|c|}{ Abbreviations } \\
\hline ACCORD & $\begin{array}{l}\text { Action to Control Cardiovascular Risk in } \\
\text { Diabetes }\end{array}$ \\
\hline $\begin{array}{l}\text { ADDITION } \\
\text { study }\end{array}$ & $\begin{array}{l}\text { Anglo-Danish-Dutch Study of Intensive } \\
\text { Treatment in People with Screen Detected } \\
\text { Diabetes in Primary Care }\end{array}$ \\
\hline $\begin{array}{l}\text { ADDQoL } \\
\text { DTSQ }\end{array}$ & $\begin{array}{l}\text { Audit of Diabetes-Dependent Quality of Life } \\
\text { Diabetes Treatment Satisfaction } \\
\text { Questionnaire }\end{array}$ \\
\hline EQ-5D & Euroqol 5 Dimensions \\
\hline EQ-VAS & Euroqol Visual Analogue Scale \\
\hline IT & Intensive treatment \\
\hline
\end{tabular}

PROMs Patient-reported outcome measures

$\mathrm{RC} \quad$ Routine care

SF-36 36-Item Short Form Health Survey

UKPDS UK Prospective Diabetes Study

W-BQ12 12-Item short form of the Well-Being

Questionnaire

\section{Introduction}

The intensive multifactorial treatment of hyperglycaemia, hypertension and dyslipidaemia halves the risk of cardiovascular events among patients with long-standing diabetes mellitus and microalbuminuria $[1,2]$. The optimal treatment for individuals identified earlier in the disease trajectory, however, remains unclear [3]. Intensive multifactorial treatment might be burdensome for patients who are largely asymptomatic. Physicians may be reluctant to introduce intensive treatment (IT) including recommendations to change multiple lifestyle behaviours and the prescription of several medications, which may lead to psychosocial stress and reduced satisfaction with treatment [4].

Patient-reported outcome measures (PROMs) are a valuable complement to outcomes such as mortality and cardiovascular events when assessing the effectiveness of early treatment. PROMs reflect patients' assessment of their own health and well-being and involve their response to questions on themes such as physical functioning, social functioning and mental well-being. They may include both generic and disease-specific questions.

PROMs are increasingly used as key performance indicators in chronic illness. Their reliability is similar to that of clinical measures such as diastolic BP or blood glucose [5], and the use of PROMs has been recommended in the evaluation of healthcare services and in regulatory decision-making [6]. Their routine use provides an opportunity to help drive changes in how healthcare is organised and delivered [5]. The UK Prospective Diabetes Study (UKPDS) suggested that IT for raised blood glucose levels and BP among British patients newly diagnosed with type 2 diabetes mellitus was not associated with adverse effects on quality of life [7]. The Action to Control Cardiovascular Risk in Diabetes (ACCORD) trial of intensive glycaemic control in US patients with long-standing type 2 diabetes mellitus did not lead to an increase in healthrelated quality of life but was associated with modest improvements in satisfaction with diabetes treatment [8].

The effects of intensive multifactorial treatment on PROMs among people with type 2 diabetes mellitus detected by screening are not known. The Anglo-Danish-Dutch Study of Intensive Treatment in People with Screen Detected Diabetes in Primary Care (ADDITION-Europe) was a pragmatic cluster-randomised trial that took place in 343 general 
practices in Denmark, England and the Netherlands to study the effects of intensive multifactorial treatment compared with routine care $(\mathrm{RC})$ in 3,057 screen-detected patients aged 40-69 years. The prescription of glucose-lowering, antihypertensive and lipid-lowering drugs increased in both groups. After 5 years of follow-up, more patients in the IT group than in the $\mathrm{RC}$ group were being prescribed aspirin, $\mathrm{ACE}$ inhibitors or angiotensin receptor blockers, glucose-lowering drugs, antihypertensive drugs and lipid-lowering drugs. IT compared with RC was associated with a non-significant $17 \%$ relative reduction of cardiovascular mortality and morbidity [3]. We now report the effects of intensive multifactorial treatment compared with RC on self-reported health status, well-being, diabetes-specific quality of life and treatment satisfaction.

\section{Methods}

\section{Design}

The full details of the design and rationale have previously been reported [3, 9-12]. In brief, ADDITION-Europe consisted of two phases: (1) a screening phase, and (2) a pragmatic, clusterrandomised parallel-group trial in four centres (Denmark, Cambridge UK, the Netherlands and Leicester UK). In total, 343 general practices were randomised to screening plus $\mathrm{RC}$ of diabetes (the RC group), or to screening followed by intensive multifactorial treatment (the IT group). Randomisation was done in a 1:1 ratio by statisticians in each centre, according to a computer-generated list, independent of measurement teams. Population-based stepwise screening programmes among people aged 40-69 years (50-69 years in the Netherlands) without known diabetes were undertaken between April 2001 and December 2006 [13, 14]. Individuals were diagnosed with type 2 diabetes mellitus according to the WHO criteria [15]. Patients were excluded if they had an illness with a life expectancy of less than 12 months, were housebound, pregnant or lactating, or had psychological or psychiatric problems that were likely to invalidate informed consent. In total, 3,057 eligible participants were enrolled (Denmark 1,533, Cambridge UK 867, the Netherlands 498, and Leicester UK 159). The study was approved by local ethics committees in each centre, and the participants provided informed consent.

\section{Intervention}

The interventions to promote the intensified treatment differed slightly in each centre [3, 9-12]. Treatment targets and algorithms were, however, common for the IT group in all centres and included $\mathrm{HbA}_{1 \mathrm{c}}<7.0 \%$ (53 mmol/mol), BP $\leq 135 /$ $85 \mathrm{mmHg}$, cholesterol $<5 \mathrm{mmol} / \mathrm{l}$ in the absence of a history of coronary heart disease and $<4.5 \mathrm{mmol} / \mathrm{l}$ in patients with a cardiovascular disease, and the prescription of aspirin to those treated with antihypertensive medication. In people with known cardiovascular diseases or a BP above 120/80 mmHg, an ACE inhibitor was recommended. After publication of the Heart Protection Study, the treatment algorithm recommended the prescription of a statin to all patients with a cholesterol concentration of $\geq 3.5 \mathrm{mmol} / 1$ [16]. Further details are available on the study website (www.addition.au.dk/, accessed 10 July 2013). In the RC group, patients with screen-detected diabetes received the standard pattern of diabetes care with less strict targets according to the national guidelines in their respective countries [17-19].

Measurements and outcomes

Anthropometric and biochemical measurements All measures were undertaken following standard operating procedures by centrally trained staff unaware of the study group allocation. Biochemical measures were analysed in five regional laboratories, without any systematic differences in the $\mathrm{HbA}_{1 \mathrm{c}}$ measures [13]. Participants were followed for a mean of 5.7 years.

Questionnaires At baseline, standardised self-report questionnaires were used to collect information on education, employment, ethnicity, lifestyle habits (smoking status and alcohol consumption), prescribed medication and health status (Euroqol 5 Dimensions [EQ-5D]). As the participants were screen-detected, no diabetes-specific measures were obtained at baseline. At follow-up, the following questionnaires were used to cover both generic and diabetes-specific measures. Questionnaires were completed at the same health assessment visit as the anthropometric and biochemical measurements.

Health status was assessed using the 36-item Short Form Health Survey (SF-36), which generates a profile of scores on eight dimensions of health: (1) physical functioning; (2) role limitations caused by physical problems; (3) social functioning; (4) bodily pain; (5) general mental health; (6) role limitations due to emotional problems; (7) vitality; and (8) general health perceptions. Two summary scales can be calculated: the physical component score and the mental component score. For all dimensions, an average score can be calculated, with a range from 0 (least favourable health state) to 100 (most favourable health state) [20].

The EQ-5D is a generic questionnaire consisting of a classification system (EQ-5D Profile) and a Visual Analogue Scale (EQ-VAS). The EQ-5D Profile was completed by participants at baseline and follow-up; the EQ-VAS was completed only at follow-up. The EQ-5D Profile covers five domains of health (mobility, self-care, usual activities, pain/discomfort and anxiety/depression), each with three levels of functioning: level 1, no problems; level 2, some problems; and level 3, severe problems. This results in 243 health states, with a score ranging from -0.594 to +1.00 (full health). A value of zero 
represents death; negative values imply a health state worse than death. An interaction term adjusts for an extra decrease when one of the dimensions is at the most severe level [21]. The EQ-VAS is a graded, vertical line anchored at 0 (worst imaginable health state) and 100 (best imaginable health state). The patient was asked to mark a point on the EQ-VAS that best reflected his or her actual health state [22].

General well-being was assessed with the 12-item short form of the Well-Being Questionnaire (W-BQ12), which measures different aspects of well-being of study participants, including patients with diabetes [23]. It can be scored on three subscales, each containing four items and with a score range of 0-12: negative well-being (a higher score meaning more negative well-being), energy (a higher score meaning more energy) and positive well-being (a higher score representing more positive well-being). Furthermore, a score for general well-being can be calculated [24], with a score range of 0-36 and a higher score indicating better well-being.

The Audit of Diabetes-Dependent Quality of Life (ADDQoL) is a measure of how patients perceive the impact and importance of diabetes and its treatment on quality of life [25]. We used the ADDQoL 19, which consists of 19 diabetesspecific items. For each item, the patient is asked how things would be without diabetes, with a score ranging from -3 (a great deal better) to 1 (worse), and then asked to rate the importance of this item, scores ranging from 3 (very important) to 0 (not at all important). A weighted rating per item can be calculated by multiplying the unweighted rating by the importance rating. The total ADDQoL score is the mean of all the weighted ratings of the applicable domains, and ranges from -9 (the maximum negative impact of diabetes) to 3 (the maximum positive impact of diabetes).

The Diabetes Treatment Satisfaction Questionnaire (DTSQ) is recommended for measuring patients' satisfaction with their diabetes treatment [26]. It consists of a six-item scale assessing treatment satisfaction and two items assessing the perceived frequency of hyperglycaemia and hypoglycaemia. The treatment satisfaction score can range from 0 (very dissatisfied) to 36 (very satisfied).

\section{Statistical analyses}

The analysis plan was finalised prior to preparation of the endpoint dataset (http://www.addition.au.dk/, accessed 10 July 2013). We compared the baseline characteristics of surviving participants who did and who did not complete both the baseline and 5-year questionnaires. For normally distributed continuous variables, we calculated means and SDs, and for non-normal variables we presented medians and 25 th and 75 th percentiles. For categorical variables, we
Fig. 1 Practice and participant flows in the ADDITION-Europe trial

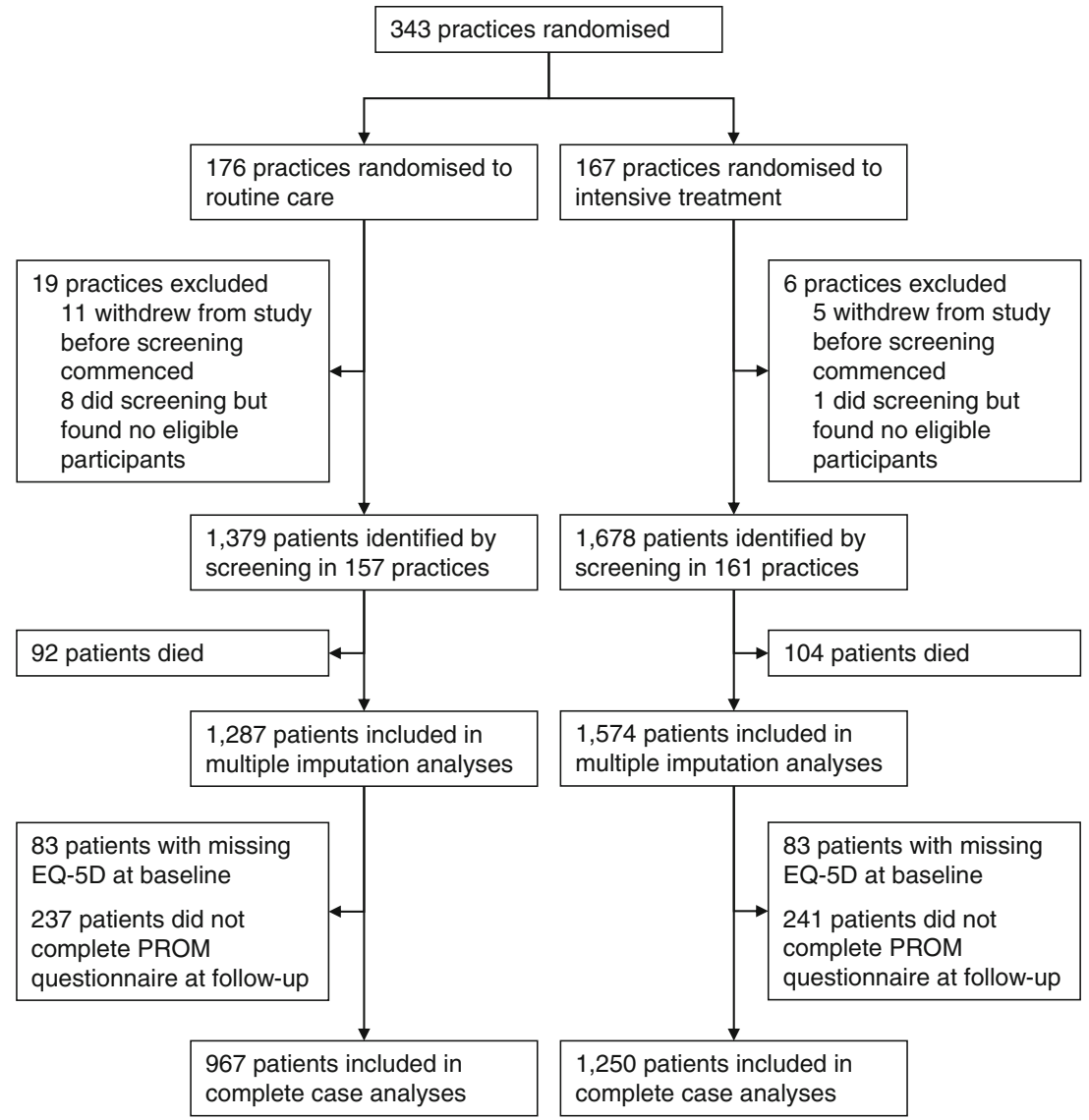


calculated the number and percentage of individuals within each category. We presented the mean scores and SDs of all PROMs at follow-up by centre and by randomised group. We used linear mixed effects regression models to estimate the difference in each PROM at follow-up, and 95\% CIs, comparing the IT group with the RC group. A random effect per general practice was included in order to account for intracluster correlation. All PROMs were left-skewed. As an alternative to transformation, we wished to control for the baseline level of the PROMs. These were not available for all measures so we included baseline EQ-5D as a proxy for baseline quality of life; correction for baseline EQ-5D greatly improved the normality of the residuals.

The estimated differences in means from the four centres were then pooled using random effects meta-analysis, and a forest plot was used to display the estimated mean differences and CIs for each centre and overall. We calculated the $I^{2}$ statistic to represent the proportion of variability between centres owing to heterogeneity.

Individuals who were lost to follow-up or did not complete both the baseline and follow-up questionnaires were excluded.
Patients with missing data might have been those who were experiencing more serious illness and greater disability. These missing data are therefore unlikely to be 'missing completely at random' but rather 'missing at random', so simply excluding these patients may lead to selection bias. Therefore, we used multiple imputation [27], imputing five datasets using patient characteristics at baseline and at the 5-year follow-up and including all patients who were alive at follow-up, to perform a sensitivity analysis.

Analyses were undertaken using SAS v9.2 (SAS Institute, Cary, NC, USA) and Review Manager v5.1 (Cochrane Collaboration, http://ims.cochrane.org/revman).

\section{Results}

Of the 343 randomised general practices, 318 (157 RC and 161 IT) completed screening and included eligible patients. At baseline, the participating practices had a prevalence of diabetes of $3.5 \%$ and nationally representative mean patient list sizes [3]. Baseline demographic, clinical, biochemical and
Table 1 Baseline characteristics of ADDITION-Europe participants who were included in the multiple imputation analysis
Data are $n(\%)$ unless otherwise specified

$\mathrm{IQR}$, interquartile range

\begin{tabular}{|c|c|c|c|c|}
\hline & $\begin{array}{l}\mathrm{RC} \\
(n=1,287)\end{array}$ & $n$ available & $\begin{array}{l}\text { IT } \\
(n=1,574)\end{array}$ & $n$ available \\
\hline \multicolumn{5}{|l|}{ Demographic variables } \\
\hline Male sex & $731(56.8 \%)$ & 1,287 & $916(58.2 \%)$ & 1,574 \\
\hline Mean (SD) age at diagnosis (years) & $60.0(6.9)$ & 1,287 & $60.1(6.9)$ & 1,574 \\
\hline White ethnicity & $1,157(92.8 \%)$ & 1,247 & $1,440(95.1 \%)$ & 1,514 \\
\hline Employed & $403(43.1 \%)$ & 935 & $465(41.7 \%)$ & 1,116 \\
\hline \multicolumn{5}{|l|}{ Clinical variables } \\
\hline History of myocardial infarction & $67(5.6 \%)$ & 1,199 & $99(6.6 \%)$ & 1,498 \\
\hline History of stroke & $20(1.7 \%)$ & 1,183 & $39(2.7 \%)$ & 1,465 \\
\hline Current smoker & $337(26.8 \%)$ & 1,257 & $405(26.1 \%)$ & 1,549 \\
\hline Median (IQR) units of alcohol /week & $5(1,12)$ & 1,105 & $4(1,12)$ & 1,399 \\
\hline Mean (SD) BMI (kg/m²) & $31.6(5.5)$ & 1,252 & $31.6(5.5)$ & 1,517 \\
\hline Mean (SD) weight (kg) & $90.3(17.3)$ & 1,254 & $91.0(17.6)$ & 1,518 \\
\hline Median (IQR) $\mathrm{HbA}_{1 \mathrm{c}}(\mathrm{mmol} / \mathrm{mol})$ & $49(43,56)$ & 1,211 & $48(43,56)$ & 1,492 \\
\hline Median (IQR) $\mathrm{HbA}_{1 \mathrm{c}}(\%)$ & $6.6(6.1,7.3)$ & 1,211 & $6.5(6.1,7.3)$ & 1,492 \\
\hline Mean (SD) systolic BP (mmHg) & $149.9(21.1)$ & 1,257 & $148.4(22.0)$ & 1,518 \\
\hline Mean (SD) diastolic BP (mmHg) & $86.8(11.2)$ & 1,257 & $86.1(11.1)$ & 1,519 \\
\hline Mean (SD) total cholesterol $(\mathrm{mmol} / \mathrm{l})$ & $5.6(1.2)$ & 1,213 & $5.5(1.1)$ & 1,498 \\
\hline Median (IQR) HDL cholesterol (mmol/l) & $1.2(1.0,1.5)$ & 1,202 & $1.2(1.0,1.5)$ & 1,476 \\
\hline Median (IQR) triacylglycerol (mmol/l) & $1.7(1.2,2.4)$ & 1,208 & $1.6(1.2,2.3)$ & 1,487 \\
\hline \multicolumn{5}{|l|}{ Self-reported medication use } \\
\hline Any antihypertensive drugs & $545(43.5 \%)$ & 1,254 & $699(46.3 \%)$ & 1,510 \\
\hline Any cholesterol-lowering drugs & $188(15.0 \%)$ & 1,254 & $258(17.1 \%)$ & 1,510 \\
\hline Aspirin & $152(12.1 \%)$ & 1,254 & $230(15.2 \%)$ & 1,510 \\
\hline \multicolumn{5}{|l|}{ Self-reported health status } \\
\hline Median (IQR) EQ-5D score & $0.85(0.73,1.00)$ & 1,204 & $0.85(0.73,1.00)$ & 1,491 \\
\hline Included in complete case analysis & $967(75.1 \%)$ & 1,287 & $1,250(79.4 \%)$ & 1,574 \\
\hline
\end{tabular}




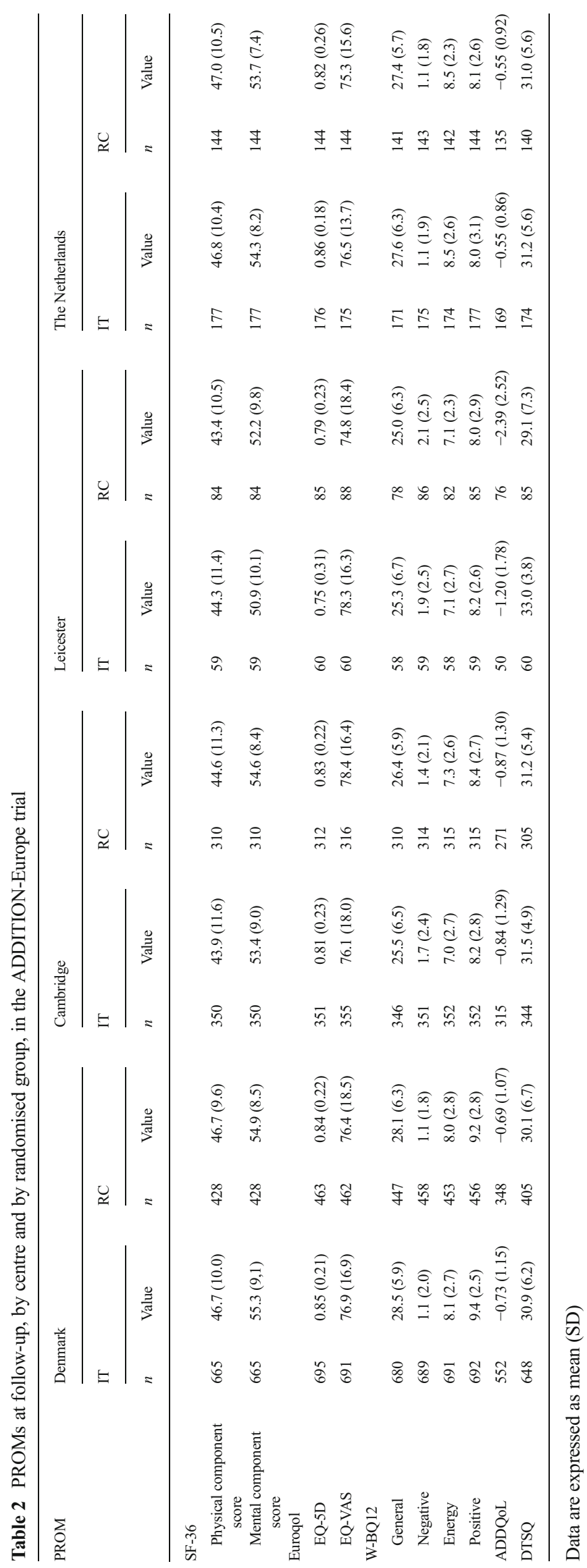


treatment characteristics of the 3,057 participants in the $\mathrm{RC}$ and IT groups were well matched overall. Their mean age at baseline was 60.3 years, $58 \%$ were male, $94 \%$ were white and $41 \%$ were employed [3].

The participant and practice flows are shown in Fig. 1. Data from the 2,861 patients included in the multiple imputation analyses are shown in Table 1 . Patients who completed the questionnaires at both baseline and follow-up $(n=2,217)$ were more likely to be male (59.5\% vs $50.8 \%$ ), of white ethnicity (95.2\% vs $89.9 \%$ ) and employed (44.8\% vs $32.7 \%$ ) than those who did not $(n=644)$. The former group were also less likely to be current smokers ( $24.3 \%$ vs $34.0 \%)$, have higher levels of alcohol consumption (median 5 units/week vs 3 units/week) and have higher EQ-5D scores at baseline (median 0.85 vs 0.81). They also had lower systolic BP (148.5 $\mathrm{mmHg}$ vs
$151.4 \mathrm{mmHg})$. Other variables were comparable between the two groups (data not shown).

EQ-5D values did not change between diagnosis and followup, with a median (interquartile range) of $0.85(0.73-1.00)$ at baseline and $0.85(0.73-1.00)$ at 5-year follow-up. Table 2 shows the PROM scores at follow-up separately for each centre and by randomised group.

The mean differences in the PROMs comparing the IT group with the RC group are shown in Figs 2, 3, 4, 5 and electronic supplementary material (ESM) Fig. 1. There were no statistically significant differences in health status (Fig. 2; for SF-36, the summary scales are presented in Fig. 2 and the eight subscales are presented in ESM Fig. 1), well-being (Fig. 3), diabetes-specific quality of life (Fig. 4) and satisfaction with diabetes treatment (Fig. 5) between the IT and RC
Fig. 2 Mean difference in health status between the IT and RC groups after 5 years of follow-up by centre (boxes) and pooled estimates (diamonds) calculated by random effects meta-analysis. The widths of the horizontal bars and diamonds denote the $95 \% \mathrm{CI}$, and the box sizes indicate the relative weight in the analysis. MCS, mental component scale; PCS, physical component scale

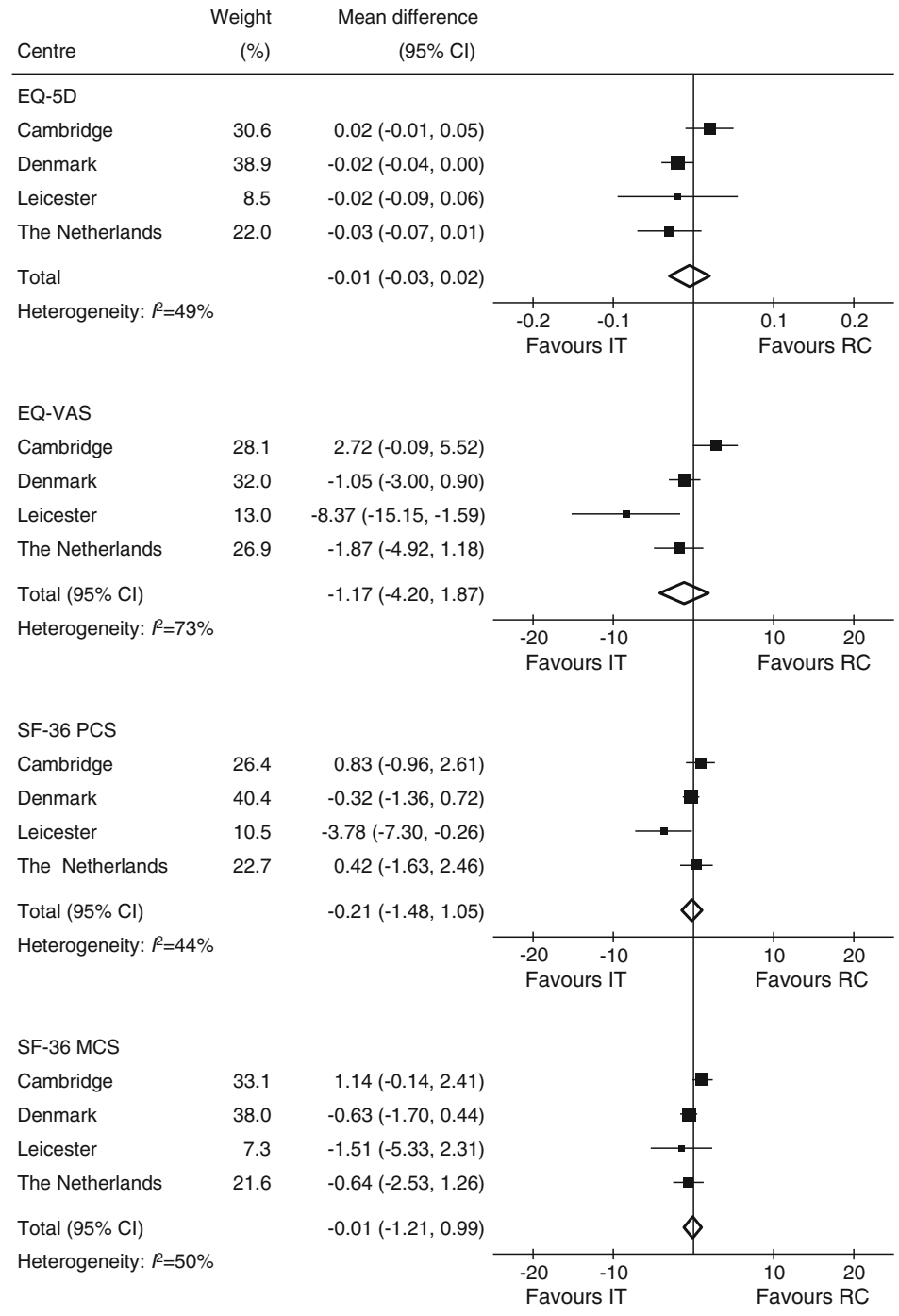


Fig. 3 Mean difference in scores from the W-BQ12 between the IT and $\mathrm{RC}$ groups after 5 years of follow-up by centre (boxes) and pooled estimates (diamonds) calculated by random effects meta-analysis. The widths of the horizontal bars and diamonds denote the $95 \% \mathrm{CI}$, and the box sizes indicate the relative weight in the analysis

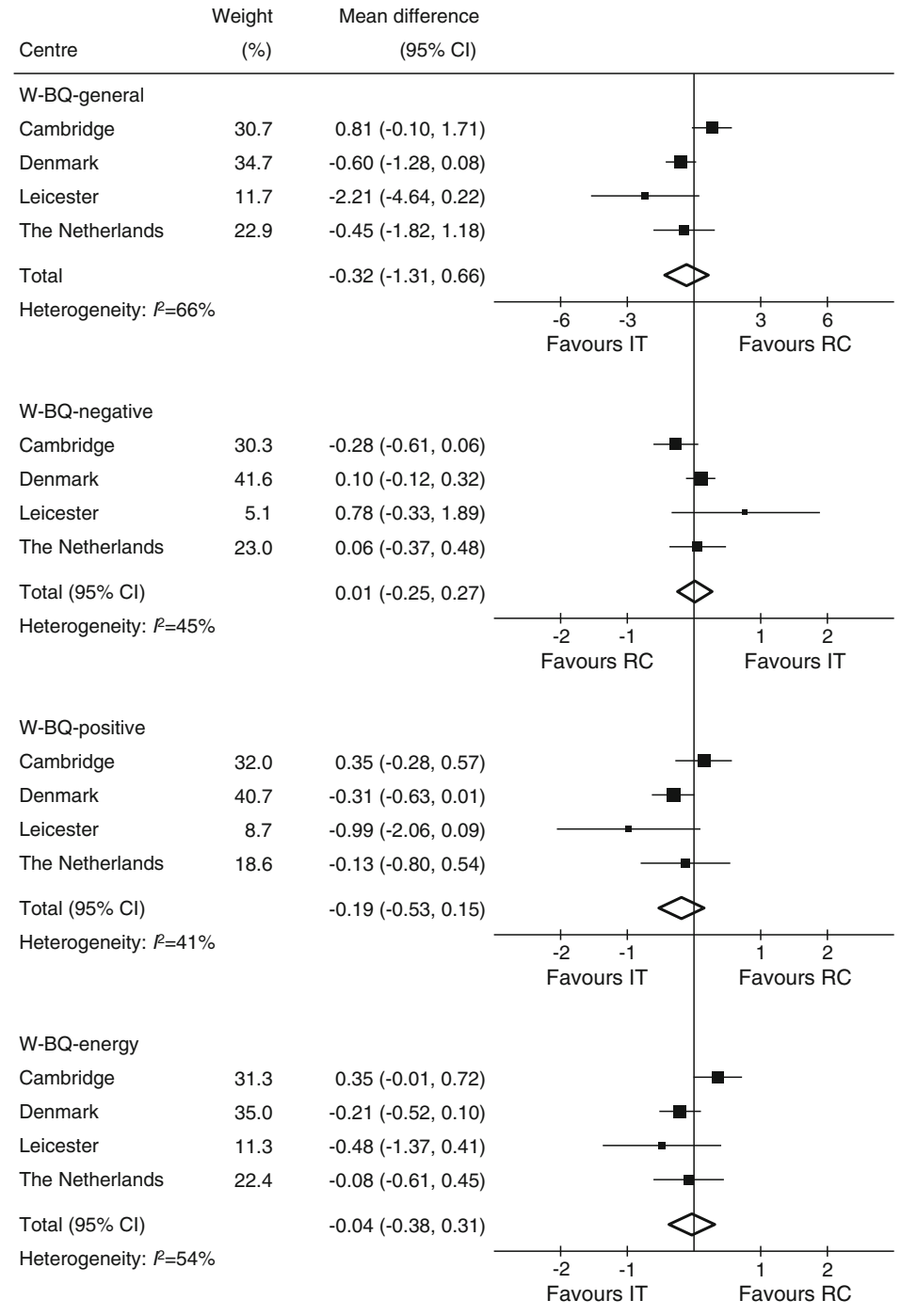

groups. There was some heterogeneity between centres, with $I^{2}$ varying between $13 \%$ (SF-36 physical functioning) and $73 \%$ (EQ-VAS).

Multiple imputation analyses resulted in slightly different point estimates, but the overall patterns were unchanged, with no statistically significant differences in any of the PROMs (results not shown).

\section{Discussion}

An intervention to promote the multifactorial target-driven, intensive management of patients with screen-detected type 2 diabetes mellitus was not associated with differences in health status, well-being, quality of life and treatment satisfaction compared with routine diabetes care after a mean follow-up of 5.7 years. Health status (as measured by the EQ-5D) did not change between diagnosis and follow-up.
The trial was undertaken during a period in which targets for BP and cholesterol levels became stricter for diabetes patients, which resulted in smaller than expected differences between the RC and IT groups, in terms of cardiovascular risk factors, prescribed medication and cardiovascular disease outcomes [3]. The differences were larger in the first years of the trial, and 1 year differences in intermediate outcomes between the RC and IT groups were not maintained at 5 years [10, 28]. As a result, the difference in PROMs between the two study groups may have been reduced. However, we also found that there were no differences in SF-36 and EQ5D scores between the treatment groups at 1 and 3 years in the Dutch arm of the trial $[10,29]$. Although pooled analyses from the four centres showed no differences overall between the IT and RC groups, for some PROMs (the SF-36 physical component scale, EQVAS, ADDQoL and DTSQ), the results from Leicester clearly favoured the IT group. This may be due to the multiethnic population in Leicester and the nature of the intervention, 
Fig. 4 Mean difference in scores from the ADDQoL questionnaire between the IT and RC groups after 5 years of follow-up by centre (boxes) and pooled estimates (diamonds) calculated by random effects meta-analysis. The widths of the horizontal bars and diamonds denote the $95 \% \mathrm{CI}$, and the box sizes indicate the relative weight in the analysis

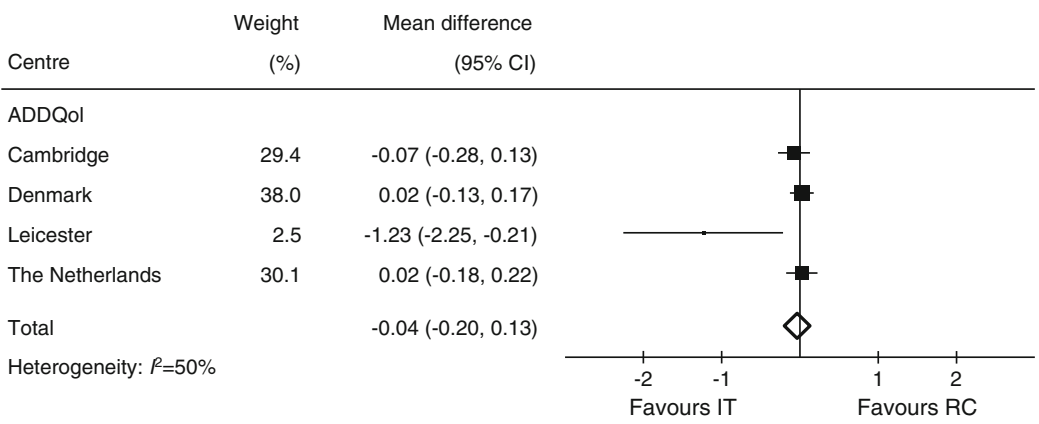

which was delivered by an intermediate care team comprising a specialist doctor, diabetes nurse educator and dietitian who were based at a community-based diabetes specialist care facility. Patients received structured education via the Diabetes Education and Self Management for Ongoing and Newly Diagnosed (DESMOND) programme [30], weight management advice and a glucometer at diagnosis. This is in contrast to other ADDITION centres, which relied more heavily upon the incumbent primary care team to achieve the targets set out in the IT protocols [28]. The different responses to intensive multifactorial treatment for type 2 diabetes mellitus immediately after screening across different populations and healthcare settings require further research.

Our study had several strengths. The sample size and follow-up of the study population were robust, and the response rate $(73 \%)$ was high. We used both generic and diabetesspecific measures: disease-specific tools are better than generic tools at quantifying temporal changes in quality of life [31]. This analysis clearly demonstrated differential effects of the intervention on PROMs across the different populations.

Our study also had some limitations. The patients who completed all the questionnaires differed from those who did not. These differences were, however small, not in one direction and similar for the IT and RC groups, so they are likely to have had only a small effect on the outcomes. The sensitivity analysis using multiple imputation confirmed the results of the main analysis, which strengthens our belief that a selective dropout of patients did not introduce significant bias. Most PROMs were not measured at baseline, which meant we were unable to investigate changes in PROMs over time. For diabetes-specific outcomes, such as the DTSQ and ADDQoL, this would not have been possible. We measured a wide range of possible confounding variables and these were similar between the groups at baseline. We would have preferred to correct each outcome for its corresponding baseline measurement, but for most PROMs no baseline measurement was available. As a proxy for baseline health status, we adjusted all models for the baseline EQ-5D level.

In contrast to other diabetes studies, the participants in ADDITION-Europe were screen-detected and hence the majority were asymptomatic. Their baseline health-related quality of life was high (median EQ-5D 0.85 on a scale with a maximum of 1.0) and remained stable over the first years of the disease, although the participants aged 5 years over the course of the study. This is an important result, particularly given the increased treatment burden over this time. In a Dutch study of 1,136 people with type 2 diabetes mellitus (mean age 64.9 years, $50 \%$ women), the average EQ-5D score was 0.74 (SD 0.27). Women, older patients and patients with a longer duration of diabetes reported lower health-related quality of life [31].

In a cluster-randomised trial in 55 Dutch primary care practices, changes in diabetes-related health status did not differ between the standard and intensive groups after 1 year of intensified multifactorial therapy, although some detrimental effects on social functioning (SF-36 social functioning showing a mean intention-to-treat difference in change between groups of -1.03 ) could not be ruled out [32]. The participants were 4 years older than the ADDITION-Europe
Fig. 5 Mean difference in scores from the DTSQ between the IT and $\mathrm{RC}$ groups after 5 years of follow-up by centre (boxes) and pooled estimates (diamonds) calculated by random effects meta-analysis. The widths of the horizontal bars and diamonds denote the $95 \% \mathrm{CI}$, and the box sizes indicate the relative weight in the analysis

\begin{tabular}{|c|c|c|c|c|}
\hline Centre & $\begin{array}{r}\text { Weight } \\
(\%)\end{array}$ & $\begin{array}{r}\text { Mean difference } \\
\qquad(95 \% \mathrm{Cl})\end{array}$ & & \\
\hline \multicolumn{5}{|l|}{ DTSQ } \\
\hline Cambridge & 34.1 & $-0.29(-1.15,0.56)$ & & \\
\hline Denmark & 32.9 & $-0.99(-1.90,-0.08)$ & & \\
\hline Leicester & 7.3 & $-4.39(-7.52,-1.25)$ & $\rightarrow$ & \\
\hline The Netherlands & 25.8 & $-0.40(-1.63,0.84)$ & & \\
\hline Total & & $-0.85(-1.76,0.07)$ & & \\
\hline \multirow{2}{*}{\multicolumn{3}{|c|}{ Heterogeneity: $R=56 \%$}} & $\begin{array}{ll}-6 & -3\end{array}$ & 36 \\
\hline & & & Favours IT & Favours $\mathrm{RC}$ \\
\hline
\end{tabular}


participants, were largely white and had a mean duration of diabetes of 6 years. Their baseline EQ-5D values were 0.82 (intervention group) and 0.84 (control group).

The UKPDS reported that intensified treatment of blood glucose and BP in patients newly diagnosed with diabetes mellitus did not adversely affect their health status [7]. In the ACCORD sub-study, participants were about 2 years older than the ADDITION-Europe patients, had a different ethnic background and had had clinically diagnosed type 2 diabetes mellitus for 9-10 years; in addition, 35\% were on insulin treatment. The change over 4 years in the SF-36 physical component score and the DTSQ scale differed significantly between the standard and intensive glucose-lowering treatment groups. However, the absolute net difference in the physical component score was only 0.5 units, far below a general threshold of three to five points for a minimally important difference of these measures [8]. Other results are largely in line with ADDITION-Europe findings, which means that there were no clinically significant differences in PROMs between treatment groups.

We have found that, following detection by screening, intensive multifactorial treatment over 5 years was not associated with differences in health status, well-being, quality of life or treatment satisfaction compared with RC. While it remains uncertain whether early IT is associated with a reduction in cardiovascular endpoints, we find no evidence that such treatment adversely affects patient-reported outcomes early in the course of the disease.

Acknowledgements We thank all patients, practice nurses and general practitioners for their contributions to the ADDITION-Europe study. We thank Ronald P. Stolk, and Bruce H.R. Wolffenbuttel, University Medical Centre Groningen, University of Groningen, the Netherlands, for participation on the steering committee that designed the ADDITION-Europe study. Bio-Rad provided equipment for $\mathrm{HbA}_{1 \mathrm{c}}$ testing during the screening phase.

Funding ADDITION-Cambridge was supported by the Wellcome Trust (grant reference no. G061895), the Medical Research Council (grant reference no. G0001164), the NIHR Health Technology Assessment Programme (grant reference no. 08/116/300), National Health Service R\&D support funding (including the Primary Care Research and Diabetes Research Networks) and the National Institute for Health Research. SJG receives support from the Department of Health NIHR Programme Grant funding scheme (RP-PG-0606-1259). The views expressed in this publication are those of the authors and not necessarily those of the UK National Health Service, National Institute of Health Research or the Department of Health. ADDITION-Denmark was supported by the National Health Services in the counties of Copenhagen, Aarhus, Ringkøbing, Ribe and South Jutland in Denmark, the Danish Council for Strategic Research, the Danish Research Foundation for General Practice, Novo Nordisk Foundation, the Danish Centre for Evaluation and Health Technology Assessment, the diabetes fund of the National Board of Health, the Danish Medical Research Council and the Aarhus University Research Foundation. The trial has been given unrestricted grants from Novo Nordisk AS, Novo Nordisk Scandinavia AB, Novo Nordisk UK, ASTRA Denmark, Pfizer Denmark, GlaxoSmithKline Pharma Denmark, Servier Denmark A/S and
HemoCue Denmark A/S. Parts of the grants from Novo Nordisk Foundation, Danish Council for Strategic Research and Novo Nordisk were transferred to the other centres. ADDITION-Leicester was supported by the Department of Health, the NIHR Health Technology Assessment Programme (grant reference no. 08/116/300), National Health Service R\&D support funding (including the Primary Care Research and Diabetes Research Network, and LNR CLAHRC) and the National Institute for Health Research. MJD and KK receive support from the Department of Health NIHR Programme Grant funding scheme (RPPG-0606-1272), NIHR Biomedical Research Unit in Nutrition, Diet and Lifestyle (BRU 2011-20113) and NIHR Collaboration for Leadership in Applied Heath Research and Care and Leicester Clinical Trials Unit. ADDITION-Netherlands was supported by unrestricted grants from Novo Nordisk, Glaxo Smith Kline and Merck, and by the Julius Center for Health Sciences and Primary Care, University Medical Center, Utrecht.

Duality of interest TL has received unrestricted grants in the period 2001 through 2011 for the ADDITION study from public foundations and the medical industry: Novo Nordisk AS, Novo Nordisk Scandinavia AB, ASTRA Denmark, Pfizer Denmark, GlaxoSmithKline Pharma Denmark, SERVIER Denmark A/S and HemoCue Denmark A/S. TL has given three lectures for the medical industry within the past 2 years and holds shares in Novo Nordisk. SJG has been reimbursed by Eli Lilly for membership of a data-monitoring and ethics committee. The other authors declare that there is no duality of interest associated with their involvement in this manuscript.

Contribution statement TL is Chair and KB-J Vice-Chair of the steering committee, which also included the following people, who collectively designed the study: SJG, AS, KK, MJD, NJW and GEHMR. SJG, AS, TL, KK, MJD, KB-J, NJW and GEHMR are principal investigators for the trial and, along with MvdD and RKSi, participated in the acquisition of the data. RKSt and MvdD carried out the statistical analyses. All authors participated in the analysis and interpretation of data. MvdD and GEHMR drafted the report. All authors participated in the critical revision of the intellectual content of the report, and all authors approved the final version.

Access to research materials Data are not currently available for public release.

Open Access This article is distributed under the terms of the Creative Commons Attribution Noncommercial License which permits any noncommercial use, distribution, and reproduction in any medium, provided the original author(s) and the source are credited.

\section{References}

1. Gaede P, Vedel P, Larsen N, Jensen GV, Parving HH, Pedersen O (2003) Multifactorial intervention and cardiovascular disease in patients with type 2 diabetes. N Engl J Med 348:383-393

2. Gaede P, Lund-Andersen H, Parving HH, Pedersen O (2008) Effect of a multifactorial intervention on mortality in type 2 diabetes. N Engl J Med 358:580-591

3. Griffin SJ, Borch-Johnsen K, Davies MJ et al (2011) Effect of early intensive multifactorial therapy on 5-year cardiovascular outcomes in individuals with type 2 diabetes detected by screening (ADDITION-Europe): a cluster-randomised trial. Lancet 378:156-167

4. Peyrot M, Rubin RR, Lauritzen T, Snoek FJ, Matthews DR, Skovlund SE (2005) Psychosocial problems and barriers to improved diabetes management: results of the Cross-National 
Diabetes Attitudes, Wishes and Needs (DAWN) Study. Diabet Med 22:1379-1385

5. Black N (2013) Patient reported outcome measures could help transform healthcare. BMJ 346:f167

6. Darzi A (2008) Quality and the NHS next stage review. Lancet 371:1563-1564

7. UK Prospective Diabetes Study Group (1999) Quality of life in type 2 diabetic patients is affected by complications but not by intensive policies to improve blood glucose or blood pressure control (UKPDS 37). Diabetes Care 22:1125-1136

8. Anderson RT, Narayan KM, Feeney P et al (2011) Effect of intensive glycemic lowering on health-related quality of life in type 2 diabetes: ACCORD trial. Diabetes Care 34:807-812

9. Echouffo-Tcheugui JB, Simmons RK, Williams KM et al (2009) The ADDITION-Cambridge trial protocol: a clusterrandomised controlled trial of screening for type 2 diabetes and intensive treatment for screen-detected patients. BMC Public Health 9:136

10. Janssen PG, Gorter KJ, Stolk RP, Rutten GE (2009) Randomised controlled trial of intensive multifactorial treatment for cardiovascular risk in patients with screen-detected type 2 diabetes: 1-year data from the ADDITION Netherlands study. Br J Gen Pract 59:43-48

11. Lauritzen T, Griffin S, Borch-Johnsen K, Wareham NJ, Wolffenbuttel BH, Rutten G (2000) The ADDITION study: proposed trial of the cost-effectiveness of an intensive multifactorial intervention on morbidity and mortality among people with type 2 diabetes detected by screening. Int $\mathrm{J}$ Obes Relat Metab Disord 24(Suppl 3):S6-S11

12. Webb DR, Khunti K, Srinivasan B et al (2010) Rationale and design of the ADDITION-Leicester study, a systematic screening programme and randomised controlled trial of multi-factorial cardiovascular risk intervention in people with type 2 diabetes mellitus detected by screening. Trials 11:16

13. Sandbaek A, Griffin SJ, Rutten G et al (2008) Stepwise screening for diabetes identifies people with high but modifiable coronary heart disease risk. The ADDITION study. Diabetologia 51:1127-1134

14. Van den Donk M, Sandbaek A, Borch-Johnsen K et al (2011) Screening for type 2 diabetes. Lessons from the ADDITIONEurope study. Diabet Med 28:1416-1424

15. Alberti KGMM, Zimmet PZ (1999) Definition, diagnosis and classification of diabetes mellitus and its complications. World Health Organization, Geneva

16. Collins R, Armitage J, Parish S, Sleigh P, Peto R (2003) MRC/BHF Heart Protection Study of cholesterol-lowering with simvastatin in 5963 people with diabetes: a randomised placebo-controlled trial. Lancet 361:2005-2016

17. McIntosh A, Hutchinson A, Home PD et al (2001) Royal College of General Practitioners. Clinical guidelines and evidence review for type 2 diabetes: management of blood glucose. University of Sheffield, Sheffield, UK
18. Royal College of General Practitioners in Denmark (1999) Type 2-diabetes in general practice-diagnosis and treatment. Royal College of General Practitioners, Copenhagen, Denmark

19. Rutten GEHM, Verhoeven S, Heine RJ et al (1999) Dutch College of General Practitioners. Practice guidelines: diabetes mellitus type 2. Huisarts Wetenschap 42:67-84

20. Ware JE Jr, Sherbourne CD (1992) The MOS 36-item short-form health survey (SF-36). I. Conceptual framework and item selection. Med Care 30:473-483

21. Dolan P (1997) Modeling valuations for EuroQol health states. Med Care 35:1095-1108

22. Brooks R (1996) EuroQol: the current state of play. Health Policy 37:53-72

23. Pouwer F, van der Ploeg HM, Ader HJ, Heine RJ, Snoek FJ (1999) The 12-item well-being questionnaire. An evaluation of its validity and reliability in Dutch people with diabetes. Diabetes Care 22:2004-2010

24. Pouwer F, Snoek FJ, van der Ploeg HM, Ader HJ, Heine RJ (2000) The well-being questionnaire: evidence for a three-factor structure with 12 items (W-BQ12). Psychol Med 30:455-462

25. Bradley C, Todd C, Gorton T, Symonds E, Martin A, Plowright R (1999) The development of an individualized questionnaire measure of perceived impact of diabetes on quality of life: the ADDQoL. Qual Life Res 8:79-91

26. Bradley C (1994) Diabetes treatment satisfaction questionnaire. In: Bradley C (ed) Handbook of psychology and diabetes: a guide to psychological measurement in diabetes research and practice. Harwood Academic, Chur, pp 111-132

27. Donders AR, van der Heijden GJ, Stijnen T, Moons KG (2006) Review: a gentle introduction to imputation of missing values. J Clin Epidemiol 59:1087-1091

28. Webb DR, Khunti K, Gray LJ et al (2012) Intensive multifactorial intervention improves modelled coronary heart disease risk in screendetected type 2 diabetes mellitus: a cluster randomized controlled trial. Diabet Med 29:531-540

29. Van den Donk M, Gorter KJ, Rutten GE (2010) No negative effects of a multi-factorial, intensified treatment on self-reported health status, treatment satisfaction, and diabetes-related distress in screendetected type 2 diabetes patients. The ADDITION-Netherlands study. Qual Life Res 19:509-513

30. Davies MJ, Heller S, Skinner TC et al (2008) Effectiveness of the Diabetes Education and Self Management for Ongoing and Newly Diagnosed (DESMOND) programme for people with newly diagnosed type 2 diabetes: cluster randomised controlled trial. BMJ 336:491-495

31. Redekop WK, Koopmanschap MA, Stolk RP, Rutten GE, Wolffenbuttel BH, Niessen LW (2002) Health-related quality of life and treatment satisfaction in Dutch patients with type 2 diabetes. Diabetes Care 25:458-463

32. Cleveringa FG, Minkman MH, Gorter KJ, van den Donk M, Rutten GE (2010) Diabetes Care Protocol: effects on patient-important outcomes. A cluster randomized, non-inferiority trial in primary care. Diabet Med 27:442-450 